\title{
Longitudinal changes in axial length in high myopia: a 4-year prospective study
}

\author{
Min Woo Lee, ${ }^{1,2}$ Seong-Eun Lee, ${ }^{1}$ Hyung-Bin Lim, ${ }^{1}$ Jung-Yeul Kim (i) ${ }^{1}$
}

${ }^{1}$ Department of Ophthalmology, Chungnam National University Hospital, Daejeon, Korea, Republic of

${ }^{2}$ Department of Ophthalmology, Konyang University College of Medicine, Daejeon, Korea, Republic of

\section{Correspondence to} Professor Jung-Yeul Kim, Department of Ophthalmology, Chungnam National University Hospital, Daejeon 301 721, Korea, Republic of; kimjy@cnu. ac.kr

Received 22 May 2019 Revised 25 June 2019 Accepted 30 July 2019 Published Online First 13 August 2019
Check for updates

(C) Author(s) (or their employer(s)) 2020. No commercial re-use. See rights and permissions. Published by BMJ.

To cite: Lee MW, Lee S-E,

Lim H-B, et al.

Br J Ophthalmol

2020;104:600-603.

\section{ABSTRACT}

Aim To determine the longitudinal changes in the axial length $(A L)$ in patients with high myopia without any other ophthalmic disease

Methods Participants were divided into two groups: a high myopia group (60 eyes) without myopic degeneration, such as chorioretinal atrophy or posterior staphyloma, and a control group (60 eyes). Both groups were further divided into subgroups according to the AL: subgroup 1 ( $\geq 27.5 \mathrm{~mm}$ ), subgroup 2 (26.0$27.5 \mathrm{~mm})$, subgroup $3(24.5-26.0 \mathrm{~mm})$ and subgroup 4 $(<24.5 \mathrm{~mm})$. The ALs were measured five times at 1-year interval using an IOL master, and the AL was fitted with linear mixed models.

Results In the high myopia group, the AL showed a relatively constant increase at each visit, and they were significantly different with previous measurements at most visits, whereas the control group showed no significant change of AL. Subgroups 1,2 and 3 showed significant changes in AL over time $(0.064,0.032$ and $0.012 \mathrm{~mm} / \mathrm{y}$, respectively). In univariate analyses, age, best-corrected visual acuity, baseline AL and anterior chamber depth were significantly correlated with changes in the AL in the high myopia group. In multivariate analysis, only baseline AL remained significant $(p<0.001)$.

Conclusions Myopic eyes, including moderately myopic eyes, showed a consistent increase in AL over 4 years, and eyes with a longer baseline AL showed a greater increase in $\mathrm{AL}$ than eyes with a shorter AL.

\section{INTRODUCTION}

Myopia is often regarded as a benign disorder because vision can be corrected with glasses or contact lens. However, it is an important public health problem in eastern and southeastern Asian countries, including Singapore, China, Taiwan, Japan and Korea, due to its increasing prevalence in the past 50-60 years. ${ }^{12}$ Especially high myopia, which is defined as a spherical equivalent (SE) $\leq-6.0$ dioptres (D), can cause various retinal disorders. Chorioretinal atrophy, choroidal neovascularisation and macular retinoschisis can occur as the structure of a highly myopic eye becomes thinner due to fewer cross-linkages. ${ }^{3}$ The Tajimi study in Japan found that myopic macular degeneration was the leading cause of unilateral and bilateral blindness. $^{4}$ Additionally, pathological myopia was the most common cause of low vision and blindness in a Chinese population aged $\geq 40$ years. ${ }^{5}$

These conformational changes are closely related to the axial length (AL) of highly myopic eyes; thus, observation of change in the $\mathrm{AL}$ is clinically important. Larsen et $a l^{6}$ reported that the growth of the AL of the eye was found to reach adult length by 13 years of age in 1852 eyes with refractive errors of \pm 5 D. However, in high myopia, many studies have reported that the AL continues to increase. ${ }^{78}$ Saka et $a l^{8}$ reported that the highly myopic eyes with a longer baseline $\mathrm{AL}$ showed a greater increase in $\mathrm{AL}$, suggesting the possibility that more myopic eyes became more myopic with increasing age. In their study, there were patients with posterior staphyloma about 32.4\% among high myopia. Although it is not clear whether these structural abnormalities affected the change of AL, they may be intervening factors for identifying the effect of the degree of AL on its changes.

Therefore, to identify the association of baseline AL with subsequent changes therein in detail, we evaluated the longitudinal changes in patients with high myopia without structural changes, such as chorioretinal atrophy or posterior staphyloma, by comparing their eyes with those of normal individuals.

\section{METHODS \\ Patients}

This prospective, longitudinal, observational study was approved by the Institutional Review Board of Chungnam National University Hospital, Daejeon, Republic of Korea, and adhered to the tenets of the Declaration of Helsinki. Patients who visited our retina clinic were enrolled and examined between May 2011 and September 2018, and informed consent was obtained from all patients. The high myopia group consisted of eyes with an $\mathrm{AL} \geq 26.0 \mathrm{~mm}$, and the control group consisted of eyes with an SE between +3.0 and $-6.0 \mathrm{D}$, and $\mathrm{AL}<26.0 \mathrm{~mm}$. To observe the effect of the degree of $\mathrm{AL}$ on $\mathrm{AL}$ changes, high myopia and control groups were divided into subgroups: subgroup 1 ( $\geq 27.5 \mathrm{~mm})$, subgroup $2(26-27.5 \mathrm{~mm})$, subgroup 3 (24.5-26 mm) and subgroup $4(<24.5 \mathrm{~mm})$, respectively. In all, 30 eyes were included in each subgroup, for a total of 60 eyes per group. All subjects underwent a complete ophthalmic examination, which included detailed ophthalmoscopic examinations, best-corrected visual acuity (BCVA), intraocular pressure (IOP) using non-contact tonometry, SE, and keratometry. Participants were followed every year for 4 years. Exclusion criteria were a BCVA $<20 / 25$, IOP $\geq 21 \mathrm{~mm} \mathrm{Hg}$, any ophthalmic disease except high myopia, a history of intraocular surgery including cataract extraction and cataract surgery during follow-up period. We also excluded eyes with high myopia including 
conformational abnormalities, such as chorioretinal atrophy or posterior staphyloma, which could affect AL changes, to observe the association of baseline AL with longitudinal changes therein in more detail.

The IOL Master (V.5.02, Carl Zeiss, Jena, Germany) was used for measuring the AL, anterior chamber depth and radius of corneal curvature. The measurements made by the IOL Master were repeated five times at each examination by a skilled examiner, and the average value was used in the statistical analyses.

\section{Statistical analyses}

Ocular parameters in the high myopia and control groups were compared using an independent t-test. To compare the AL measurements among timepoints, a paired t-test was used. $P$ values were adjusted using the Bonferroni correction. Linear mixed models were performed to identify the rate of AL change in each subgroup. The AL was fitted with linear mixed models with time, age, IOP, sex, keratometry, initial anterior chamber depth and radius of corneal curvature as fixed effects. A random intercept was included at the eye level. Univariate and multivariate generalised linear mixed models were used to determine the factors associated with AL changes over time in the high myopia group. Statistical analyses were performed using SPSS V.18.0 software (IBM Corp., Armonk, NY, USA).

\section{RESULTS}

\section{Demographics and AL measurements at each visit}

Of the 126 eyes, four eyes in the high myopia group and two eyes in the control group were excluded because they underwent cataract surgery during the follow-up period. Overall, 60 eyes of 60 participants in each group were enrolled. The mean age of the high myopia and control groups were $40.6 \pm 13.4$ years (range, $20-58$ years) and $42.4 \pm 13.4$ years (range, $21-57$ years), respectively; they were not significantly different $(\mathrm{p}=0.494)$ (table 1).

Sex, BCVA, IOP and anterior chamber depth were also not significantly different. The average SE, AL, keratometry and radius of corneal curvature of the high myopia and control groups were significantly different, at $-8.47 \pm 4.00 \mathrm{D}$ versus $-1.73 \pm 2.06 \mathrm{D}$ $(\mathrm{p}<0.001), 28.19 \pm 1.79 \mathrm{~mm}$ versus $24.67 \pm 0.99 \mathrm{~mm}(\mathrm{p}<0.001)$ $42.31 \pm 2.03$ versus $43.24 \pm 1.82(\mathrm{p}=0.015)$, and $7.99 \pm 0.40 \mathrm{~mm}$ versus $7.82 \pm 0.34 \mathrm{~mm}(\mathrm{p}=0.023)$, respectively.

Table 1 Demographic characteristics of the patients at the initial examination

\begin{tabular}{lllr}
\hline & $\begin{array}{l}\text { High myopia group } \\
(\mathbf{n}=60)\end{array}$ & $\begin{array}{l}\text { Control group } \\
(\mathbf{n}=60)\end{array}$ & P value \\
\hline Age (years, mean \pm SD) & $40.6 \pm 13.4$ & $42.4 \pm 13.4$ & $0.494^{*}$ \\
\hline Sex (males, \%) & $39(66.1 \%)$ & $36(60.0 \%)$ & $0.639 \dagger$ \\
SE (diopters, median, IQR) & $-8.00(-10.75$ to -6.00$)$ & $-1.50(-3.25$ to -0.25$)$ & $<0.001^{*}$ \\
BCVA (logMAR, mean $\pm S D)$ & $-0.05 \pm 0.23$ & $-0.05 \pm 0.08$ & $0.961^{*}$ \\
IOP (mm Hg, mean $\pm S D)$ & $15.6 \pm 2.2$ & $14.9 \pm 2.4$ & $0.102^{*}$ \\
\hline Keratometry (mean $\pm S D)$ & $42.31 \pm 2.03$ & $43.24 \pm 1.82$ & $0.015^{*}$ \\
\hline Axial length (mm, median, & $27.48(27.05$ to 29.17$)$ & $24.55(24.19$ to 25.54$)$ & $<0.001^{*}$ \\
IQR) & & & \\
\hline ACD (mm, mean $\pm S D)$ & $3.73 \pm 0.46$ & $3.61 \pm 0.40$ & $0.136^{*}$ \\
\hline RCC (mm, mean $\pm S D)$ & $7.99 \pm 0.40$ & $7.82 \pm 0.33$ & $0.023^{*}$ \\
\hline
\end{tabular}

Values in bold face $(\mathrm{p}<0.05)$ are statistically significant.

* Independent t-test.

$+\chi^{2}$ test.

$A C D$, anterior chamber depth; BCVA, best-corrected visual acuity; IOP, intraocular pressure

$\mathrm{RCC}$, radius of corneal curvature; $S E$, spherical equivalent; logMAR, logarithm of the minimum angle of resolution.
Table 2 AL at each visit in the high myopia and control groups

\begin{tabular}{lllll} 
& High myopia group & P value & Control group & P value \\
\hline Baseline AL & $28.19 \pm 1.79$ & & $24.67 \pm 0.99$ & \\
First year & $28.22 \pm 1.81$ & 0.397 & $24.68 \pm 0.98$ & 0.153 \\
Second year & $28.27 \pm 1.83$ & $<0.001$ & $24.71 \pm 0.99$ & 0.001 \\
Third year & $28.30 \pm 1.86$ & 0.012 & $24.72 \pm 1.00$ & 0.055 \\
Fourth year & $28.36 \pm 1.87$ & $\mathbf{0 . 0 0 2}$ & $24.72 \pm 1.01$ & 0.825 \\
\hline
\end{tabular}

Values in boldface $p<0.013$ after Bonferroni correction are statistically significant. All values are expressed as the mean \pm SD $(\mathrm{mm})$.

Paired t-test was used for difference with previous measurement.

$A L$, axial length.

In the high myopia group, the AL showed a relatively constant and typically significant increase versus previous visit (table 2).

The control group did not show a significant AL changes at most visits. The high myopia and control groups did not show a significant difference in SE between first and last visits $(-8.26 \pm 4.01 \mathrm{D}$ vs $-8.43 \pm 4.98 \mathrm{D}, \mathrm{p}=0.761 ;-1.73 \pm 2.06 \mathrm{D}$ vs $-1.41 \pm 2.59 \mathrm{D}, \mathrm{p}=0.881$, respectively).

\section{The rates of $A L$ changes in each subgroup}

The rates of AL change in the high myopia and control groups were 0.047 and $0.011 \mathrm{~mm} /$ years, respectively. Subgroup 1 $(\geq 27.5 \mathrm{~mm})$ and subgroup $2(26.0-27.5 \mathrm{~mm})$, which are the high myopia subgroups, showed a noticeable increase in the AL over time (figure 1). However, subgroup $3(24.5-26.0 \mathrm{~mm})$ and subgroup $4(<24.5 \mathrm{~mm})$ did not show significant changes over time when compared with the high myopia subgroups. The rate of AL change in subgroup 1 was $0.064 \mathrm{~mm} / \mathrm{y}$, which was statistically significant $(\mathrm{p}<0.001)$ (table 3$)$.

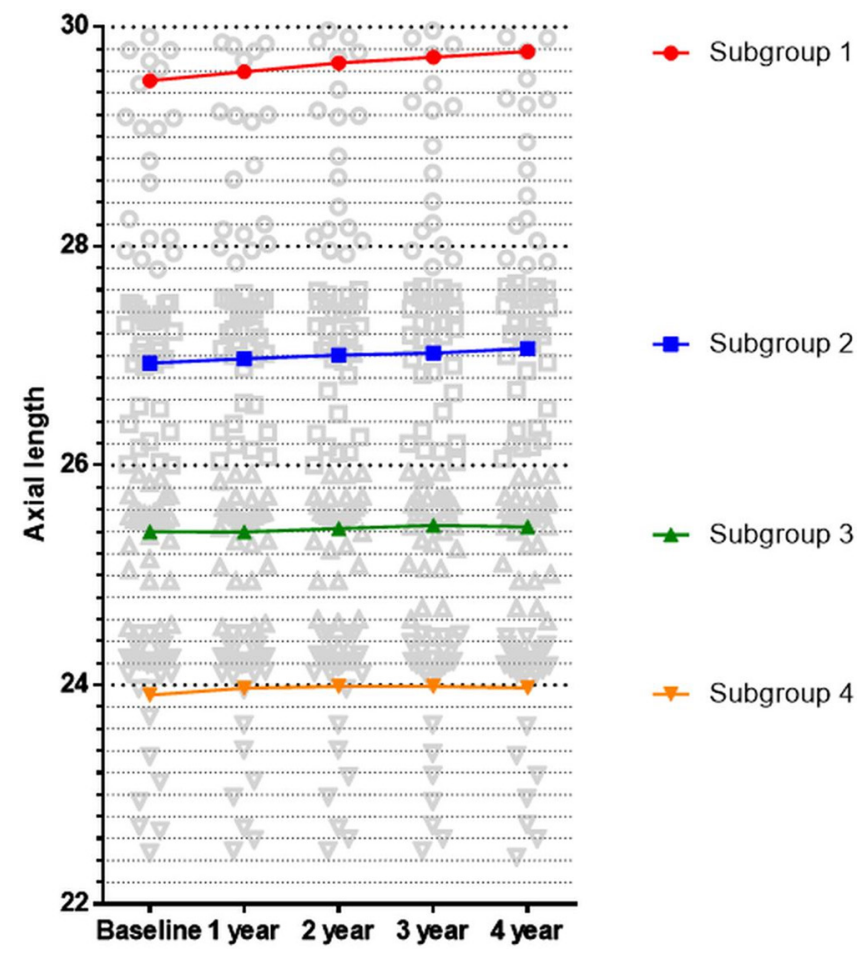

Figure 1 Scatterplots and line graphs showing mean AL by subgroup. Subgroups with longer baseline AL showed more definite increases in AL over time: subgroup 1 ( $\geq 27.5 \mathrm{~mm})$, subgroup 2 (26.0-27.5 mm), subgroup $3(24.5-26.0 \mathrm{~mm})$, and subgroup 4 (<24.5 mm). AL, axial length. 
Table 3 Rate of change in axial length in each subgroup calculated using linear mixed models

\begin{tabular}{llc}
\hline Subgroup & Rate of change & P value $^{*}$ \\
\hline Subgroup 1 $(95 \% \mathrm{Cl})(\mathrm{mm} / \mathrm{y})$ & $0.064(0.050,0.079)$ & $<0.001$ \\
Subgroup 2 $(95 \% \mathrm{Cl})(\mathrm{mm} / \mathrm{y})$ & $0.032(0.022,0.042)$ & $<0.001$ \\
Subgroup 3 $(95 \% \mathrm{Cl})(\mathrm{mm} / \mathrm{y})$ & $0.012(0.002,0.025)$ & 0.025 \\
Subgroup 4 $(95 \% \mathrm{Cl})(\mathrm{mm} / \mathrm{y})$ & $0.006(-0.001,0.012)$ & 0.054 \\
\hline
\end{tabular}

Subgroup 1: $\geq 27.5 \mathrm{~mm}$, subgroup 2: $26.0-27.5 \mathrm{~mm}$, subgroup 3: $24.5-26 \mathrm{~mm}$, subgroup $4:<24.5 \mathrm{~mm}$.

*Values in boldface $(\mathrm{p}<0.05)$ are significant changes over time in linear mixed models.

The rates of AL changes in subgroup 2 and 3 were $0.032 \mathrm{~mm} / \mathrm{y}$ and $0.012 \mathrm{~mm} / \mathrm{y}$, respectively; they were also statistically significant $(p<0.001$ and $p=0.025$, respectively). The rate of change in subgroup 4 was $0.006 \mathrm{~mm} / \mathrm{y}$, which was non-significant $(\mathrm{p}=0.054)$.

\section{Factors affecting AL changes in high myopia}

Univariate analyses showed that age, BCVA, baseline AL and anterior chamber depth were significantly associated with AL changes $(p=0.045, p=0.005, p<0.001$ and $p=0.006$, respectively) (table 4).

\section{DISCUSSION}

Highly myopic eyes can occur many kinds of conformational changes over time. Choudhury et $a l^{9}$ reported that myopic degeneration such as tessellation, peripapillary atrophy, posterior staphyloma or lacquer cracks was more prevalent among older patients. Numa et $a l^{10}$ reported that the mean and variance of fundus curvature increased with age, suggesting that the shape of the staphyloma was steeper and less smooth in elderly subjects. Our previous study also found that highly myopic eyes had a significantly greater decrease in the peripapillary retinal nerve fibre layer, and the reduction rate was greater in older patients. ${ }^{11}$ Such changes can cause visual impairment in patients with high myopia, so clinicians should observe those things carefully. Additionally, these changes are known to be associated with AL in previous studies. ${ }^{7912}$ Therefore, those myopic degenerations could be predicted to some extent by measuring the AL and changes therein.

Our study enrolled a high myopia group without signs of myopic degeneration, such as chorioretinal atrophy or posterior

\begin{tabular}{|c|c|c|c|c|}
\hline & \multicolumn{2}{|l|}{ Univariate } & \multicolumn{2}{|l|}{ Multivariate } \\
\hline & Estimate $(95 \% \mathrm{Cl})$ & $P$ value & Estimate $(95 \% \mathrm{Cl})$ & $P$ value \\
\hline Age & 0.038 (0.002 to 0.074$)$ & 0.040 & $0.001(-1.262$ to 0.003$)$ & 0.942 \\
\hline Sex & -0.286 ( -1.336 to 0.765$)$ & 0.588 & & \\
\hline BCVA & $-3.011(-5.077$ to -0.946$)$ & 0.005 & $0.118(-0.037$ to 0.273$)$ & 0.133 \\
\hline Keratometry & $-0.025(-0.272$ to 0.223$)$ & 0.843 & & \\
\hline IOP & $-0.162(-0.382$ to 0.058$)$ & 0.146 & & \\
\hline Baseline AL & 1.024 (1.007 to 1.041$)$ & $<0.001$ & 1.032 (1.010 to 1.053$)$ & $<0.001$ \\
\hline Baseline ACD & 1.512 (0.452 to 2.752$)$ & 0.006 & $-0.023(-0.102$ to 0.056$)$ & 0.568 \\
\hline Baseline RCC & $0.042(-1.214$ to 1.300$)$ & 0.947 & & \\
\hline
\end{tabular}

Values in bold face $(p<0.05)$ are statistically significant.

In multivariate analyses, only baseline AL showed a significant association $(\mathrm{p}<0.001)$.

$A C D$, anterior chamber depth; $A L$, axial length; BCVA, best-corrected visual acuity; IOP, intraocular pressure; RCC, radius of corneal curvature; $\mathrm{SE}$, spherical equivalent. staphyloma, to measure AL more accurately and evaluate the association of baseline $\mathrm{AL}$ with subsequent changes therein in more detail. In patients with these signs of myopic degeneration, it can be difficult to accurately measure the AL because of fixation loss. Furthermore, Yang et $a l^{13}$ reported that posterior staphyloma could be an important factor in AL measurements because it reduces their accuracy even with good fixation. Additionally, other previous studies have reported a correlation of the AL with posterior staphyloma. ${ }^{7810}$ By excluding these intervening factors, we could measure the AL and changes therein more accurately and reliably.

In the high myopia group, keratometry and radius of corneal curvature were significantly different from the control group. Previous studies reported that there appeared to be a definite tendency towards steeper corneas in shorter eyes and flatter corneas in longer eyes, which confirmed the emmetropising relationship between the $\mathrm{AL}$ and corneal curvature. ${ }^{14} 15$ Our study showed significantly lower keratometry in the high myopia group, which was similar to previous studies. Jonas et al ${ }^{16}$ reported that a longer radius of corneal curvature was positively associated with a longer AL in young and adult populations, which is consistent with our results. Additionally, the anterior chamber depth was deeper in the high myopia group, although it was not statistically significant. Chung et $a l^{17}$ reported that the anterior chamber depth increased with increasing AL in moderately myopic eyes; however, the anterior chamber depth showed no further increase with eyeball elongation in highly myopic eyes. Other previous studies reported that high axial elongation was associated with a thinning of the sclera and choroid posterior to the equator, whereas scleral thickness in the pars plana and limbal regions did not significantly differ between axially elongated eyes and eyes with a normal AL. ${ }^{18-20}$ It seemed that the anterior chamber depth increased with increasing AL within limits; however, high axial myopia was associated with changes located mostly posterior to the equator.

The high myopia group showed a significant increase in AL at most visit, whereas the control group showed smaller and non-significant changes at most visit; this was consistent with previous studies. ${ }^{7-1012}$ Saka $\mathrm{et} \mathrm{al}^{7}$ explained this elongation of the $\mathrm{AL}$ as increased depth of the vitreous chamber, which is consistent with the previous histological studies mentioned above. ${ }^{19} 20$

Ohsugi et $a l^{21}$ reported that change in the AL in the high myopia without complication group was $0.041 \pm 0.05 \mathrm{~mm} / \mathrm{y}$. In our study, the rate of change in AL in the high myopia group was $0.047 \mathrm{~mm} / \mathrm{y}$, which is consistent with the previous study. In subgroup analyses, subgroups 1, 2 and 3 showed a significant change in AL over time, which consisted of moderate to high myopia. Additionally, there were higher rates of change in the longer AL subgroups, especially subgroup 1 ( $0.064 \mathrm{~mm} / \mathrm{y})$. Such a change in AL can cause many problems, including changes in the SE. Surgery for refractive correction, such as posterior intraocular lens implantation after clear lens extraction, phakic intraocular lens implantation or laser-assisted subepithelial keratomileusis, has been done widely in high myopia these days; many previous studies reported difficulty of accurate prediction of SE or change in refractive error after these types of surgeries in high myopia. ${ }^{22-24}$ The change in AL would add a bad influence on a refractive error over time. Further longitudinal studies for real impact of AL change to SE in high myopia are needed.

Previous studies have reported a relationship between age and AL in normal eyes. Fotedar et $a l^{25}$ reported a decrease in the mean AL with increasing age: $23.61 \mathrm{~mm}$ (95\% CI, 23.50 to $23.73 \mathrm{~mm}$ ) in 59-year to 64-year-olds and decreased to $23.15 \mathrm{~mm}$ (95\% CI, 22.83 to $23.47 \mathrm{~mm}$ ) in $\geq 85$-year olds. Jivrajka et al ${ }^{14}$ 
also reported that with increasing age, the mean $\mathrm{AL}$ decreased $(\mathrm{r}=-0.127 ; \mathrm{p}<0.001)$. Subgroup $4(\mathrm{AL}<24.5 \mathrm{~mm})$ in our study showed a $0.006 \mathrm{~mm} / \mathrm{y}$ rate of increase in the AL, which was not statistically significant $(p=0.054)$. The difference in our results to those of previous studies may have been due to the ages of the patients, who were much younger in our study. However, subgroup 3 , which included patients with mild to moderate myopia, had a $0.012 \mathrm{~mm} / \mathrm{y}$ rate of increase $(\mathrm{p}=0.025)$. Gradual elongation of the eyeball may therefore also affect patients with moderate myopia as well as patients with high myopia, although the degree of change was small, and thus of minimal clinical significance.

We analysed factors affecting AL changes in highly myopic eyes: the age, BCVA, baseline AL and anterior chamber depth were all significant in univariate analyses. In multivariate analyses, we anticipated that age and baseline AL would be significant factors; however, only the baseline AL showed a significant association $(\mathrm{p}<0.001)$. This result could be explained as follows. The mean age of our high myopia group was $40.6 \pm 13.4$ years, which was relatively younger than that of subjects in previous studies $\left(48.4 \pm 12.2\right.$ years, Saka $\mathrm{et}_{\mathrm{al}} \mathrm{l}^{8} ; 57.3 \pm 13.6$ years, Numa et $\left.a l^{10}\right)$. As the eyeball elongates over time, the age may affect the increase in AL more as time goes by. So age would affect the change of AL in relatively older patients more than younger patients. Additionally, subgroups 1,2 and 3 showed a significant increase in AL over time; however, the degree of change in subgroup 1 , which had the longest $\mathrm{AL}$, was six times greater than that in subgroup 3. Although age may be an important factor, the $\mathrm{AL}$ at baseline was the factor most strongly associated with subsequent changes therein.

\section{Study limitations and strengths}

This study had some limitations. First, our participants were relatively younger than those in previous studies, so they may not be representative of all age groups. Second, we did not analyse SE changes because the follow-up period was not enough for analyses of SE changes.

Besides the prospective design, in which there were five measurement timepoints at 1-year interval over a period of 4 years, an additional strength of our study was that it compared AL changes between patients with high myopia and normal subjects. Additionally, we divided the participants into subgroups according to the degree of $\mathrm{AL}$, to observe the association of baseline AL with subsequent changes therein in more detail. Excluding patients with myopic degeneration and including patients with good visual acuity were also strength of the present study with respect to accurately measuring the AL.

In conclusion, myopic eyes, even those with moderate myopia, showed a consistent increase in AL over 4 years. In addition, eyes with a longer AL at baseline showed a greater increase in AL than eyes with shorter baseline AL. This may help explain the positive relationship between a longer AL and various myopic changes, such as chorioretinal atrophy and posterior staphyloma. Based on the results of the present study, we suggest that physicians carefully consider AL increases in patients with high myopia, as these suggest the possibility of myopic degeneration.

Contributors Design and conduct of the study (MWL and JYK); collection of data (MWL, SEL and JYK); analysis and interpretation of data (MWL, HBL and JYK); writing the article (MWL and JYK); critical revision of the article (MWL, HBL and JYK); final approval of the article (MWL, SEL, HBL and JYK).
Funding statement The authors have not declared a specific grant for this research from any funding agency in the public, commercial or not-for-profit sectors.

Competing interests None declared.

Patient consent for publication Not required.

Provenance and peer review Not commissioned; externally peer reviewed.

Data availability statement No data are available.

ORCID iD

Jung-Yeul Kim http://orcid.org/0000-0003-3679-1310

\section{REFERENCES}

1 Pan C-W, Ramamurthy D, Saw S-M. Worldwide prevalence and risk factors for myopia. Ophthalmic Physiol Opt 2012;32:3-16.

2 Lin LLK, Shih YF, Hsiao CK, et al. Prevalence of myopia in Taiwanese school children: 1983 to 2000. Ann Acad Med Singapore 2004;33:27-33.

3 Curtin BJ, Iwamoto T, Renaldo DP. Normal and staphylomatous sclera of high myopia. An electron microscopic study. Arch Ophthalmol 1979:97:912-5.

4 Iwase A, Araie M, Tomidokoro A, et al. Prevalence and causes of low vision and blindness in a Japanese adult population: the Tajimi study. Ophthalmology 2006;113:1354-62. e1

$5 \mathrm{Xu} \mathrm{L}$, Wang Y, Li Y, et al. Causes of blindness and visual impairment in urban and rural areas in Beijing. Ophthalmology 2006;113

6 Larsen JS. The sagittal growth of the eye. IV. ultrasonic measurement of the axial length of the eye from birth to puberty. Acta Ophthalmol 1971;49:873-86.

7 Saka N, Ohno-Matsui K, Shimada N, et al. Long-Term changes in axial length in adult eyes with pathologic myopia. Am J Ophthalmol 2010;150:562-8. e1.

8 Saka N, Moriyama M, Shimada N, et al. Changes of axial length measured by IOL master during 2 years in eyes of adults with pathologic myopia. Graefes Arch Clin Exp Ophthalmol 2013;251:495-9.

9 Choudhury F, Meuer SM, Klein R, et al. Prevalence and characteristics of myopic degeneration in an adult Chinese American population: the Chinese American eye study. Am J Ophthalmol 2018;187:34-42.

10 Numa S, Yamashiro K, Wakazono T, et al. Prevalence of posterior staphyloma and factors associated with its shape in the Japanese population. Sci Rep 2018:8:4594.

11 Lee M-W, Kim J-M, Shin Y-I, et al. Longitudinal changes in peripapillary retinal nerve fiber layer thickness in high myopia: a prospective, observational study. Ophthalmology 2019;126:522-8.

12 Asakuma T, Yasuda M, Ninomiya T, et al. Prevalence and risk factors for myopic retinopathy in a Japanese population: the Hisayama study. Ophthalmology 2012;119:1760-5.

13 Yang JY, Kim HK, Kim SS. Axial length measurements: comparison of a new swept-source optical coherence tomography-based biometer and partial coherence interferometry in myopia. J Cataract Refract Surg 2017:43:328-32.

14 Jivrajka R, Shammas MC, Boenzi T, et al. Variability of axial length, anterior chamber depth, and lens thickness in the cataractous eye. J Cataract Refract Surg 2008:34:289-94.

15 Hoffer KJ. Biometry of 7,500 cataractous eyes. Am J Ophthalmol 1980;90:360-8.

16 Jonas JB, Bi HS, Wu JF, et al. Corneal curvature radius in myopia of schoolchildren versus adult myopia. Cornea 2016;35:1333-7.

17 Chung HJ, Park CK. The distinct biometric features of high myopia compared to moderate myopia. Curr Eye Res 2016;41:1580-3.

18 Olsen TW, Aaberg SY, Geroski DH, et al. Human sclera: thickness and surface area. Am J Ophthalmol 1998;125:237-41.

19 Vurgese S, Panda-Jonas S, Jonas JB. Scleral thickness in human eyes. PLoS One 2012; 7:e29692.

20 Norman RE, Flanagan JG, Rausch SMK, et al. Dimensions of the human sclera: thickness measurement and regional changes with axial length. Exp Eye Res 2010;90:277-84

21 Ohsugi H, Ikuno Y, Shoujou T, et al. Axial length changes in highly myopic eyes and influence of myopic macular complications in Japanese adults. PLoS One 2017:12:e0180851.

22 Lam JKM, Chan TCY, Ng ALK, et al. Outcomes of cataract operations in extreme high axial myopia. Graefes Arch Clin Exp Ophthalmol 2016;254:1811-7.

23 Zaldivar R, Shultz MC, Davidorf JM, et al. Intraocular lens power calculations in patients with extreme myopia. J Cataract Refract Surg 2000;26:668-74.

24 Tychsen L, Hoekel J. Refractive surgery for high bilateral myopia in children with neurobehavioral disorders: 2. laser-assisted subepithelial keratectomy (LASEK). J Aapos 2006:10:364-70.

25 Fotedar R, Mitchell P, Burlutsky $G$, et al. Relationship of 10-year change in refraction to nuclear cataract and axial length findings from an older population. Ophthalmology 2008;115:1273-8. 